\title{
A procedure to detect, construct, quantify, numerically simulate and validate fracture networks in coal blocks
}

\author{
Peng-fei He $\cdot$ Pinnaduwa H. S. W. Kulatilake • \\ Dong-qiao Liu $\cdot$ Man-chao He $\cdot$ Xin Chen
}

Received: 23 May 2016/Accepted: 14 July 2016/Published online: 25 July 2016

(C) Springer International Publishing Switzerland 2016

\begin{abstract}
Coal mass is a typical sedimentary rock mass with three approximately orthogonal discontinuity sets: bedding planes, face cleats and butt cleats. The mechanical anisotropy of coal masses is dependent on this fabric to a large extent. In this paper, a framework incorporating X-ray computed tomography (CT) scanning, statistical analysis, the fracture tensor based methodology and a numerical stress analysis technique is proposed to detect, construct, quantify and simulate the fracture networks in coal blocks. The CT scanning was first used to obtain the geometric information of the fractures in cubic coal blocks. Then a statistical numerical procedure was proposed to estimate and construct the locations, orientations and sizes of the fractures. An overall assessment of the constructed fracture networks was next made using the fracture tensor based methodology to incorporate all the important features: number of joint sets, joint density, joint orientation and joint size. The fracture networks in cubic blocks were then numerically simulated through a proposed modified
\end{abstract}

P. He · P. H. S. W. Kulatilake ( $\square)$

Rock Mass Modeling and Computational Rock Mechanics Laboratories, University of Arizona, Tucson, AZ 85721, USA

e-mail: kulatila@u.arizona.edu

P. He $\cdot$ D. Liu $\cdot$ M. He $\cdot$ X. Chen

State Key Laboratory for Geomechanics and Deep Underground Engineering, China University of Mining and Technology (Beijing), Beijing 100083, China fictitious joint procedure to accommodate a large quantity of non-persistent joints with an acceptable numerical stress analysis effort. The framework was validated at different stages to evaluate the reliability. Finally, the implementation of this framework is shown by a numerical investigation of the influence of the fracture networks and confining stresses on the jointed coal mass strength. The proposed framework provides a systematic and innovative method to tackle the fracture network induced mechanical anisotropy of the fractured rock masses.

Keywords CT scanning - Fracture tensor - Coal blocks $\cdot$ Modified fictitious joint procedure $\cdot$ Coal mass strength

\section{Introduction}

Most naturally occurring discontinuous rock masses comprise of intact rock interspaced with different types of discontinuities. The following different types of discontinuities exist in rock masses: fissures, fractures, joints, faults, bedding planes, shear zones and dykes. In this paper, discontinuity, fracture, joint are used interchangeably. Due to the presence of discontinuities, the geomechanical response of discontinuous rock masses can be highly complicated under complex geological and in situ stress systems. Two remarkable effects of the fracture networks are 
the scale effect and mechanical anisotropy. The scale effect results mainly from the combined effect of fracture density and fracture size. The representative elementary volume (REV) size is the demarcation whether the scale effect plays an important role or not. The preferred fracture orientations contribute to the mechanical anisotropy to a large extent. Based on the rock fracture data available from a dam site in China, Wu and Kulatilake (2012) investigated the REV size with respect to the fracture system as well as mechanical properties. Their research clearly indicated that the strength and deformability of rock masses are highly influenced by the pre-existing fracture system of the rock masses. Therefore, it is very important to incorporate all the essential features of the fracture networks (number of the fracture sets, fracture density, fracture orientation and fracture size) in working with jointed rock masses.

Proper consideration of the pre-existing fracture networks would not only be beneficial to the assessment of the rock mass stability as indicated above, but also be very useful for fluid flow modeling through jointed rock masses (Andersson and Dverstorp 1987; Cacus et al. 1990; Panda and Kulatilake 1999; Kulatilake and Panda 2000; Wang et al. 2002) and hydraulic fracturing studies. The hydraulic fracturing is one of the critical techniques used in the production of shale gas, coalbed methane and extraction of geothermal energy. Men et al. (2013) numerically simulated the hydraulic fracturing process in heterogeneous rock specimens using Realistic Failure Process Analysis (RFPA2D). The numerical results show that the initiation pressure, the breakdown pressure and the fracture evolution vary with the different bedding plane angles. A numerical investigation of hydraulic fracturing in an orthogonal anisotropic shale using an extended finite element method (XFEM) performed by Wang et al. (2016) indicated that the fracture propagation direction during the hydraulic fracturing process is dependent on the combined effects of the in situ stress and orthotropic fracture system of the shale formation. In a fractured rock mass, the hydraulic fracture paths may get diverted due to the interaction between the hydraulically created and pre-exiting fractures. The numerical model established by Damjanac and Cundall (2016) based on the synthetic rock mass (SRM) concept shows that the hydraulic fractures evolve in terms of both intact rock fracturing and sliding and opening of pre-existing joints.

\section{Brief literature review on fracture observations, quantification and modeling}

Because of the intrinsic discontinuous characteristic of rock masses, observing of geometrical features of fractures from either boreholes or exposed rock faces is a fundamental work for all scales of rock mechanics problems. The ISRM suggested a general procedure for borehole based fracture observations (Li et al. 2012). The following fracture mapping techniques using traditional tools have been reported in the literature to record the exposed fractures on the rock faces: the linear scanline sampling (Priest and Hudson 1981; Kulatilake et al. 2003; Priest 1993) and rectangular window sampling (Baecher 1980; Pahl 1981; Kulatilake and Wu 1984; Priest 1993). Many efforts have been spent to incorporate remote mapping techniques into areal sampling method: photogrammetry (Hagan 1980; Harrison 1993; Coe 1995), total station (Bulut and Tüdeş 1996) and laser scanning (Slob et al. 2005; Feng and Röshoff 2015; Kulatilake and Shu 2015). In the laboratory scale, the fracture networks can be detected at a more accurate level in the rock mass specimens using X-Ray Computed Tomography (CT) scanning technique. Based on the fracture observations, many different rock mass classification systems have been proposed to evaluate the rock mass and quantify the influence of the fracture networks. Edelbro (2003) and Edelbro et al. (2006) reviewed twenty-one different rock mass classification systems developed for different scenarios. Because of the inadequate considerations of some fracture features, these scalar based evaluation systems seem to be insufficient to capture the influences of the fracture networks especially with respect to mechanical anisotropy and scale effects. The fabric tensor concept (Oda 1982) has been extended in terms of the directional fracture tensor component and applied in the fractured rock mass studies in estimating mechanical and hydraulic properties by Kulatilake et al. (1993), Wu and Kulatilake (2012), Kulatilake and Panda (2000) and Wang et al. (2002). This fracture tensor based methodology is capable of incorporating the important fracture features: number of the fracture sets, fracture density, fracture size and fracture orientation. Based on the fracture observation data, discrete fracture networks (DFN) can be created. The DFN based numerical modeling has been used to study the strength and deformability of a fractured rock mass at the two-dimensional level using UDEC by Bidgoli et al. 
(2013) and Bidgoli and Jing (2014). Wu and Kulatilake (2012) built up a DFN at the three dimensional level for a selected rock mass at the Yujian River Dam site in China using a software package developed by Kulatilake et al. (2003). The developed DFN was then incorporated into 3DEC to perform a numerical investigation on strength and deformability of the selected rock mass capturing both mechanical anisotropy and scale effects at the three-dimensional level. The DFNs obtained from the field data are established based on certain probability distributions. In this paper, attempts are made to incorporate the real fracture networks obtained through CT scanning into coal blocks. The scope of this paper is limited to coal masses which is a typical sedimentary rock mass having three approximately orthogonal discontinuity sets (bedding plane, face cleat and butt cleat). This work started from fracture detection in $110 \mathrm{~mm}$ side dimension cubic coal blocks using an industrial CT scanner. The fracture networks were then constructed based on the CT images and quantified by the fracture tensor based methodology. Finally, a modified fictitious joint framework was proposed to build up the geometric model of the cubic coal blocks having a large quantity of real fractures aiming at reducing the numerical stress analysis calculation efforts.

\section{Detection, construction, and quantification of fracture networks}

Similar to the CT scanning in medical image diagnosis, industrial CT scanning technique also uses irradiation (most frequently X-rays) to create cross sections of the targets. The 3-D structures of the targets can be constructed from the cross-sectional images (virtual 'slices'). As a non-destructive technique, the industrial CT scanning has been applied in many areas such as: detection of flaws and cavities, analyses of pores and morphology of objects, failure analysis, assembly analysis and reverse engineering applications.

\subsection{CT scanning tests}

The industrial CT scanning facility ACTIS300-320/ 225 available at the State Key Laboratory of Coal Resources and Safe Mining, CUMTB is used to observe the fractures that exist in $110 \mathrm{~mm}$ side dimension cubic coal blocks. The schematic diagram of the CT scanning process is shown in Fig. 1. The generator shoots X-ray beam at the rock specimen placed on a turntable. After penetrating the specimen, the X-rays continue to transmit and are received by the detector. The information obtained from the detector is recorded by the controlling and processing center for data processing, image processing and display. The industrial CT scanners can be categorized into two main types: line/fan beam or cross-sectional CT scanners and cone beam or volume CT scanners. The cone beam scanning method used in this research is shown in the schematic diagram Fig. 2.

Twenty-four $110 \mathrm{~mm}$ side dimension coal cubes were subjected to the cone beam CT scanning. Through data processing by Matlab software package, the raw data can be converted to hundreds of matrices. Each matrix is composed of the gray-scale value of each point on the same cross section. The gray-scale value varies from 0 (pure black: void) to 255 (pure

Fig. 1 Schematic diagram of the CT scanning process

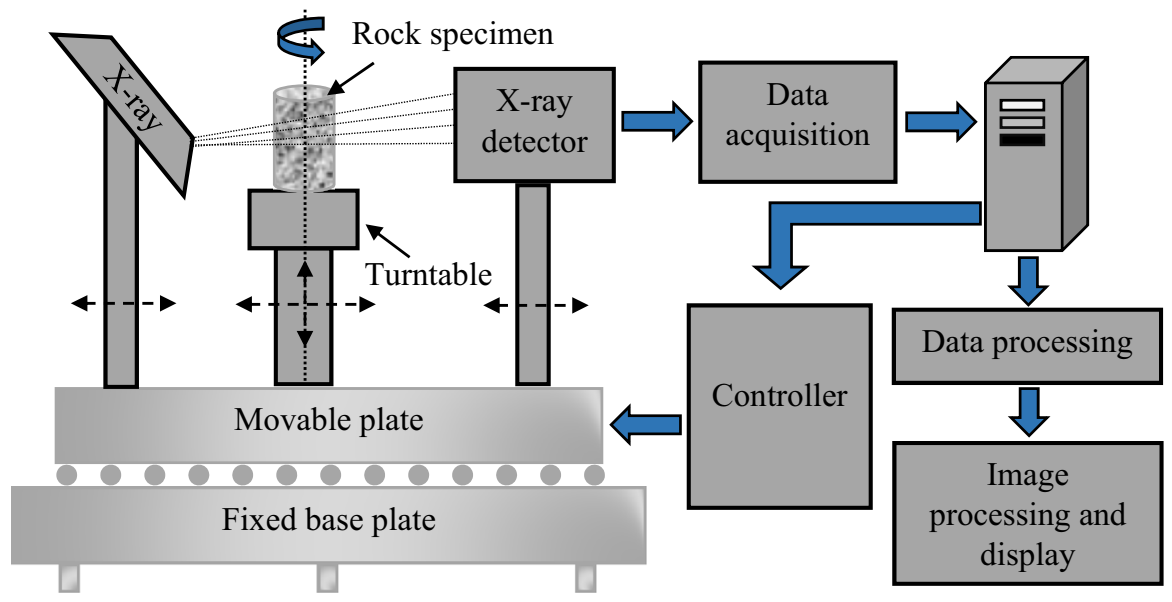


Fig. 2 Schematic diagram of the cone beam or volume CT scanners

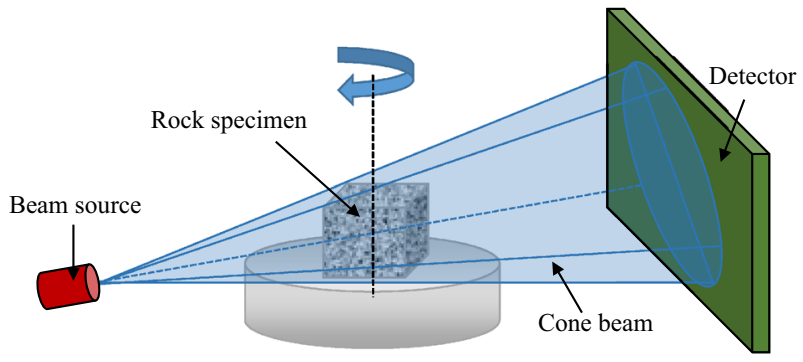

Fig. 3 Construction of the vertical CT slices approximately perpendicular to the bedding planes: a Schematic diagram of construction. b An example of a completed construction

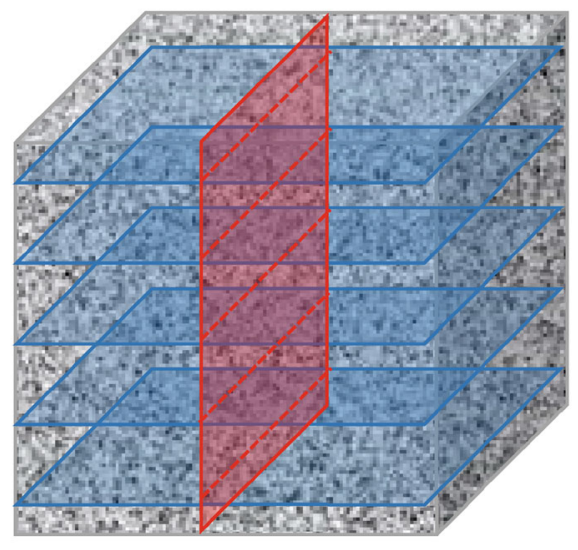

(a)

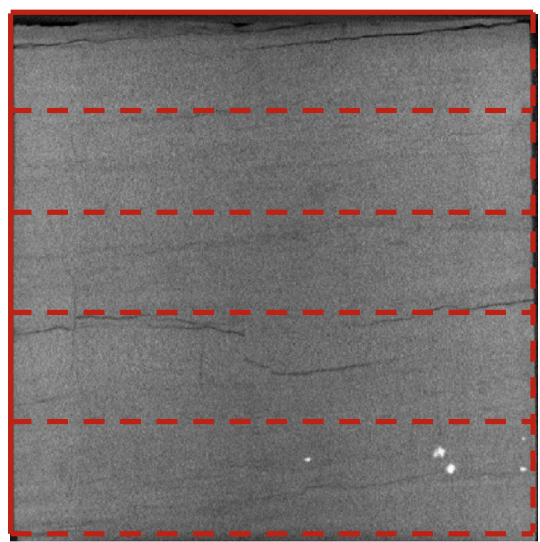

(b) white: solid). Through image processing, these matrices can be displayed as gray-scale images of the cross sections at different positions. Because of the specific experimental setup of the cubical coal blocks, the CT slices first obtained are subparallel to the bedding planes spaced at approximately $0.125 \mathrm{~mm}$, the so called horizontal CT slices. These horizontal CT slices are only capable of detecting the face cleats and butt cleats which are usually approximately perpendicular to the bedding planes. To capture the bedding planes, the vertical CT slices which are approximately perpendicular to the bedding planes are required. The same rows or columns of the aforementioned matrices can be extracted and rearranged into new matrices to represent the vertical CT slices (see Fig. 3a). These new matrices are used to construct the vertical CT slices through a similar image processing procedure as horizontal slices. A constructed example of the vertical CT slices is shown in Fig. 3b). To validate the reliability of these constructed horizontal and vertical CT slices, the CT slices closest to the faces of the block were compared with the photographs of the corresponding faces in
Fig. 4. Faces $\mathrm{A}$ and $\mathrm{C}$ are approximately perpendicular to the bedding planes whereas faces $\mathrm{E}$ and $\mathrm{F}$ are subparallel to the bedding planes. Figure 4 shows that the CT slices match quite well with the photographs of the corresponding faces. This indicates that the constructed CT slices are reliable to be used to construct the fracture networks in the coal cubes.

\subsection{A procedure to construct fracture networks}

A procedure is developed to construct the pre-existing fracture networks in the cubic coal blocks using the horizontal and vertical CT slices. The nomenclatures and assumptions of the proposed procedure are first explained as follows.

\subsubsection{Nomenclatures}

(a) Strike or strike angle: the horizontal angle measured clockwise from the north direction to the strike line, which is the intersection of the discontinuity with the horizontal plane; (b) Dip direction: the azimuth of the horizontal trace of the dip line, 


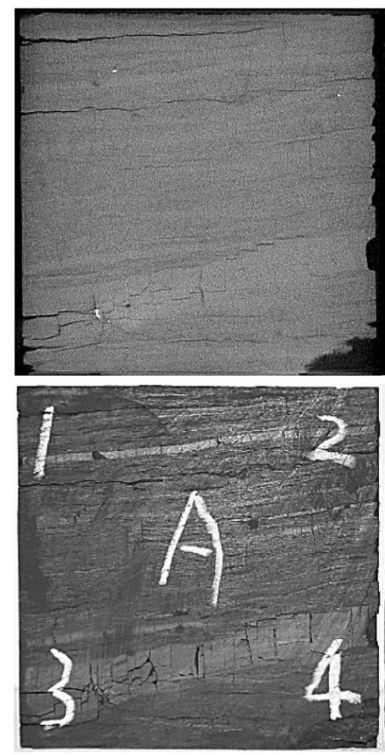

(a)

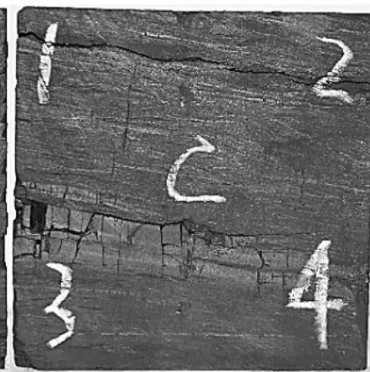

(b)

Fig. 4 Comparison between the CT slices and photographs of the corresponding faces of CB40 sample as an example: a Face $A$ and corresponding CT slice. b Face $C$ and corresponding CT

measured clockwise from the north; (c) Dip angle: the dihedral angle the fracture plane makes with the horizontal plane, which gives the steepest angle of descent of a discontinuity plane to a horizontal plane. It is measured in the vertical plane perpendicular to the strike; (d) Apparent dip angle: the vertical angle of a discontinuity plane measured in a vertical plane not perpendicular to the strike. The orientation or attitude of a geological feature is illustrated in Fig. 5.

\subsubsection{Assumptions}

Let the spacing of the CT slices used for the fracture network constructions be $\mathrm{T}$; (a) If more than one trace are observed from the CT slices, the fracture is extended $\mathrm{T} / 2$ backward from the $\mathrm{CT}$ slice where it first appears and $\mathrm{T} / 2$ forward from the CT slice where it finally disappears as long as the extended part does not exceed the block surface; (b) If only one trace is available from the CT slice, the fracture is extended $\mathrm{T} / 2$ along both forward and backward from the trace unless the extension exceeds the block surface.

The construction procedure is explained as follows:

a. The traces belonging to the same fracture plane were marked starting from the CT slice where the

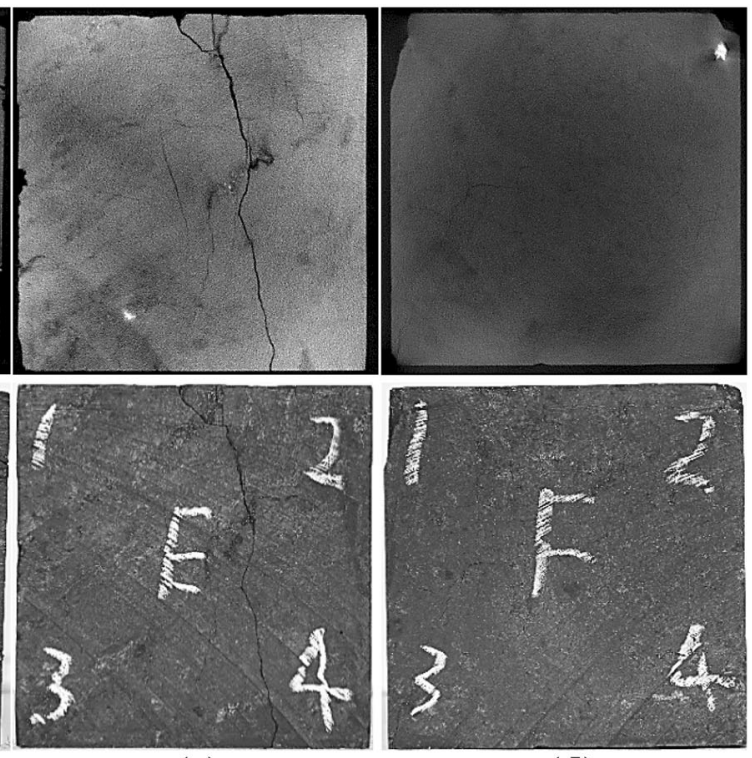

(c)

(d)

slice. c Face $E$ and corresponding CT slice. d Face $F$ and corresponding CT slice

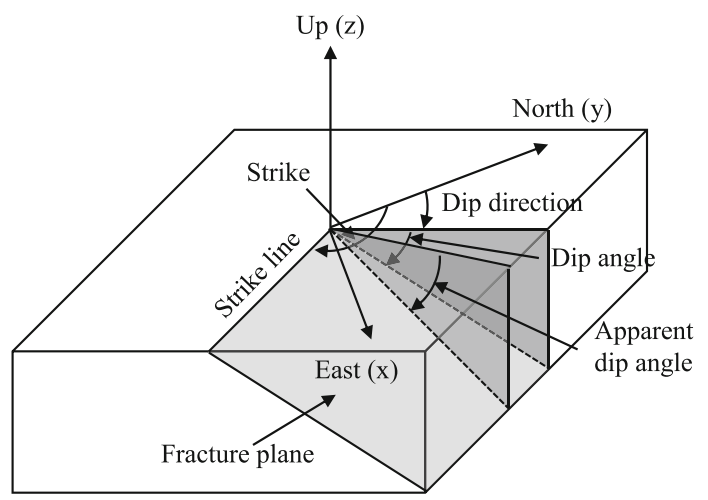

Fig. 5 Nomenclature of the orientation or attitude of a geological feature

fracture first appeared to the subsequent $\mathrm{CT}$ slices until no trace could be observed on the next CT slice. Even though the CT slices were available at an extremely close spacing $(0.125 \mathrm{~mm}$ approximately), the CT slices at a spacing of $10 \mathrm{~mm}$ were selected as a compromise between the time spent on the construction effort and accuracy;

b. On each trace, several key points were marked and their 3-D coordinates were recorded. The number of key points must be sufficient to capture the remarkable geometric changes of each trace; 
c. The 3-D coordinates were subjected to multiple linear regression analysis to obtain the equation of the fracture plane. Resulting reasonably high multiple $R$ values $(\geq 0.75)$ indicated successful fracture plane fits;

d. For the fracture planes with only one trace available from the CT slices, 2-D linear regression analysis was implemented;

e. The aforesaid equations were then used to compute the orientation of each fracture plane;

f. The irregular fracture plane was represented as a polygon. The polygon was then discretized into triangles. The area and 3-D coordinates of the center of the area $\left(G_{x}, G_{y}\right.$ and $\left.G_{z}\right)$ for each fracture plane were calculated based on the discretized triangular elements;

g. The equivalent diameter for each fracture plane was calculated based on the fracture area as a parameter to compute the fracture tensor components. The side lengths of the equivalent square or rectangle were also computed for the convenience of application in the subsequent numerical modeling.

As an example, Table 1 provides the estimated 3-D fracture geometry parameter values for some selected fractures of sample CB6 constructed from the aforementioned procedure. The fracture network containing 330 different fractures of sample CB6 constructed from the CT scans are projected on the lower hemispherical equal angle stereo-net in terms of the pole vectors using the Rocscience software package Dips 6.0 in Fig. 6. The pole vector is the normal vector of a fracture plane. From Fig. 6, the 330 individual fractures in sample CB6 can be divided into three joint sets. The mean dip angles and dip directions of the three joint sets are calculated using Dips 6.0 and listed in Table 2. The results show that the three joint sets are approximately orthogonal to each other, which reflects the intrinsic characteristics of the fracture networks of coal masses as well as the validity of the proposed construction procedure using CT scans.

\subsection{Quantification of the fracture network}

Oda (1982) proposed the concept of fracture tensor. Fracture tensor is a comprehensive index of the joint geometry parameters - joint density, orientation, joint size and the number of joint sets. By modeling joints as equivalent thin circular discs, the general form of the fracture tensor at the 3-D level for the kth joint set can be expressed as:

$\mathrm{F}_{\mathrm{ij}}^{(\mathrm{k})}=2 \pi \rho \int_{0}^{\infty} \iint_{\Omega / 2} \mathrm{r}^{3} \mathrm{n}_{\mathrm{i}} \mathrm{n}_{\mathrm{j}} \mathrm{f}(\mathrm{n}, \mathrm{r}) \mathrm{d} \Omega \mathrm{dr}$

where $i, j$ are any two axes of the selected Cartesian coordinate system, $\rho\left(\mathrm{m}^{-3}\right)$ is the joint density (which means the average number of joints per unit volume), $r$ (m) is the radius of the equivalent circular joint, $n$ is the unit normal vector of the joint plane, $n_{i}$ and $n_{j}$ are the directional cosines of vector $n$ with respect to axes $i$

Table 1 Estimated 3-D fracture geometry parameter values for some selected fractures of sample CB6 from CT scans

\begin{tabular}{|c|c|c|c|c|c|c|c|c|c|c|c|c|}
\hline $\begin{array}{l}\text { Fract. } \\
\#\end{array}$ & $R$ & $C$ & $A$ & $B$ & $\begin{array}{l}\text { Dip angle } \\
\left({ }^{\circ}\right)\end{array}$ & $\begin{array}{l}\text { Dip Dir. } \\
\left(^{\circ}\right)\end{array}$ & $G_{x}$ & $G_{y}$ & $G_{z}$ & $\begin{array}{l}\text { Area } \\
\left(\mathrm{mm}^{2}\right)\end{array}$ & $\begin{array}{l}\text { ED } \\
(\mathrm{mm})\end{array}$ & $\begin{array}{l}\text { ES } \\
(\mathrm{mm})\end{array}$ \\
\hline 1 & 0.97 & 72.16 & 0.68 & -0.02 & 89.01 & 124.29 & 80.57 & 12.50 & 5.55 & 284.39 & 19.03 & 16.86 \\
\hline 2 & 0.95 & 46.69 & -2.93 & -0.33 & 83.92 & 18.83 & 22.29 & 2.81 & 49.02 & 564.36 & 26.81 & 23.76 \\
\hline 3 & 0.98 & 41.54 & 0.76 & -0.02 & 88.92 & 127.09 & 48.69 & 11.00 & 49.46 & 478.33 & 24.68 & 21.87 \\
\hline 4 & 0.98 & 52.59 & 0.15 & 0.02 & 88.99 & 278.67 & 62.54 & 53.08 & 104.53 & 438.26 & 23.62 & 20.93 \\
\hline 5 & 0.96 & 46.61 & 0.22 & 0.14 & 82.31 & 282.45 & 63.97 & 13.20 & 104.48 & 234.76 & 17.29 & 15.32 \\
\hline 6 & 1.00 & 122.64 & -5.95 & 0.43 & 85.91 & 189.53 & 103.15 & 10.85 & 104.37 & 81.93 & 10.21 & 9.05 \\
\hline 7 & 0.99 & 38.88 & -0.07 & -0.06 & 5.46 & 48.62 & 26.43 & 75.50 & 32.22 & 172.40 & 14.82 & 13.13 \\
\hline 8 & 0.98 & 26.97 & 0.05 & -0.06 & 4.50 & 318.34 & 29.20 & 18.27 & 27.43 & 426.76 & 23.31 & 20.66 \\
\hline 9 & 0.82 & 21.14 & -0.03 & -0.02 & 2.00 & 58.68 & 27.05 & 11.51 & 20.13 & 415.58 & 23.00 & 20.39 \\
\hline 10 & 0.79 & 86.59 & 0.00 & -0.04 & 2.39 & 356.76 & 75.21 & 55.41 & 84.46 & 6598.28 & 91.66 & 81.23 \\
\hline
\end{tabular}

$R$ is the square root of the coefficient of determination in the regression analysis; $C, A, B$ are the regression coefficients of the equation obtained for the fracture plane; $G_{x}, G_{y}, G_{z}$ are $x, y, z$ coordinates of the center of the area of the fracture plane; Area is the area of the fracture plane; ED is the diameter of an equivalent circular shaped fracture; ES is the side length of an equivalent square shaped fracture 
Fig. 6 Contour plot of pole vectors of all the fractures in sample CB6 constructed from CT scans projected on equal angle stereo-net

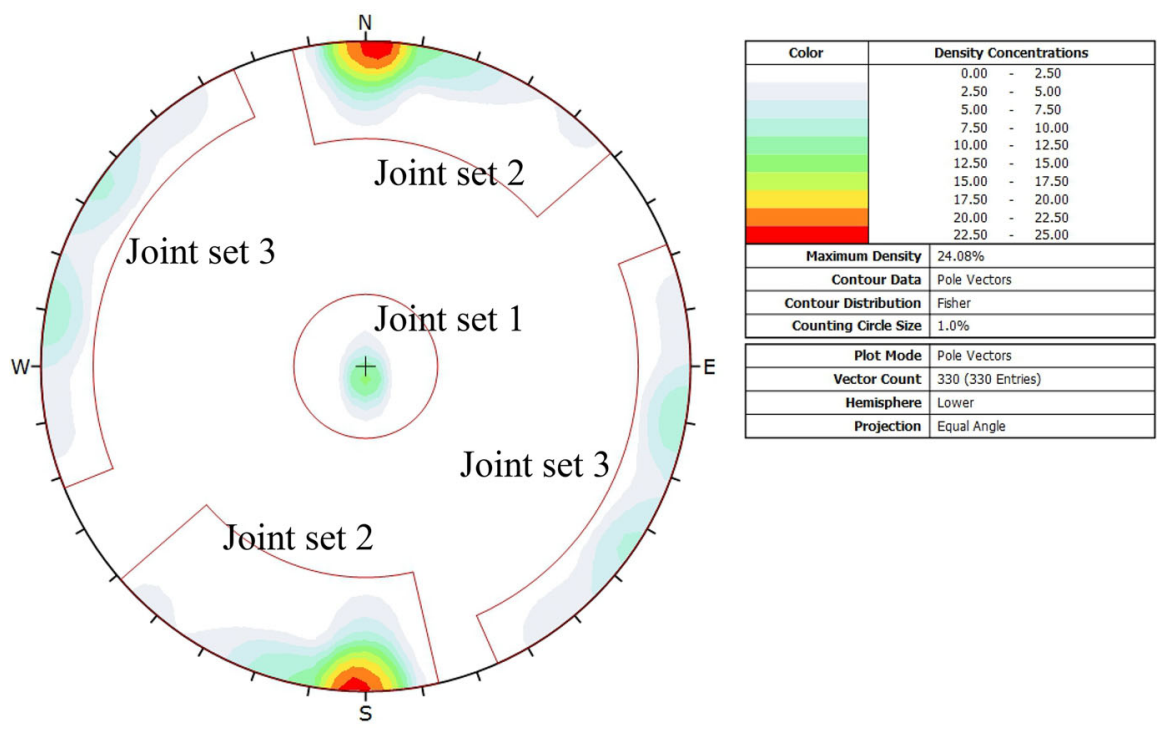

Table 2 Summary of three joint sets of sample CB6 constructed from CT scans

\begin{tabular}{|c|c|c|c|}
\hline Joint set ID & Type & Mean dip angle & Mean dip direction \\
\hline 1 & Bedding plane & 3 & 1 \\
\hline 2 & Face cleat or butt cleat & 90 & 7 \\
\hline 3 & Butt cleat or face cleat & 90 & 292 \\
\hline
\end{tabular}

and $j$ respectively, $f(n, r)$ is the joint probability density function of $n$ and $r, \Omega / 2$ is a solid angle corresponding to the surface of a unit hemisphere. If the joint orientation, $n$, and joint radius, $r$, are mutually independent, the above equation can be reduced to Eq. (2) for the fracture tensor for kth joint set:

$\mathrm{F}_{\mathrm{ij}}^{(\mathrm{k})}=2 \pi \rho \int_{0}^{\infty} \mathrm{r}^{3} \mathrm{f}(\mathrm{r}) \mathrm{dr} \iint_{\Omega / 2} \mathrm{n}_{\mathrm{i}} \mathrm{n}_{\mathrm{j}} \mathrm{f}(\mathrm{n}) \mathrm{d} \Omega$

where $f(n)$ and $f(r)$ are the probability density functions for unit normal vector $n$ and size $r$, respectively. If a rock mass has $N$ sets of joints, the fracture tensor for the rock mass can be expressed as:

$\mathrm{F}_{i j}=\sum_{\mathrm{k}=1}^{\mathrm{N}} \mathrm{F}_{i j}^{(\mathrm{k})}$

Kulatilake et al. (1993) proposed the concept of directional fracture tensor component and related this parameter to the directional deformability and strength of jointed rock masses. The directional fracture tensor component in direction $l$ for a rock mass can be expressed as:
$\mathrm{F}_{l l}=\sum_{\mathrm{k}=1}^{\mathrm{N}} \mathrm{F}_{l l}^{(\mathrm{k})}$

In the conducted study, the fracture tensor was computed using the discretized form of the fracture tensor expression given below:

$\mathrm{F}_{\mathrm{ij}}=2 \pi \rho \frac{1}{\mathrm{M}} \sum_{\mathrm{m}=1}^{\mathrm{M}} \mathrm{r}_{\mathrm{m}}^{3} \mathrm{n}_{\mathrm{i}}^{\mathrm{m}} \mathrm{n}_{\mathrm{j}}^{\mathrm{m}}$

where $M$ is the number of fracture planes in the rock mass.

Based on the above Eq. (5) and constructed fracture networks, fracture tensors for the cubical coal blocks were calculated. Table 3 shows the calculated values of the fracture tensor for ten blocks (nine components, principal values, principal direction vectors, the first invariant of the fracture tensor $I_{1}$ and the second invariant of the deviatoric fracture tensor $J_{2}$ ). $I_{1}$ indicates the porosity of the rock mass resulting from the fractures. $J_{2}$ is an index of the degree of structural anisotropy of the fracture system. The values given in Table 3 show that most $F_{x x}$ components are the 
Table 3 Calculation results of fracture tensors obtained for ten coal blocks

\begin{tabular}{|c|c|c|c|c|c|c|c|c|c|c|}
\hline Sample \# & CB1 & CB2 & CB3 & CB4 & CB5 & CB6 & CB7 & CB8 & CB9 & CB10 \\
\hline$F_{x x}$ & 0.156 & 0.142 & 0.219 & 0.023 & 0.253 & 0.507 & 0.305 & 0.101 & 0.060 & 0.419 \\
\hline$F_{x y}$ & 0.008 & 0.003 & 0.003 & 0.001 & 0.029 & 0.017 & 0.027 & 0.005 & 0.010 & 0.020 \\
\hline$F_{x z}$ & 0.018 & 0.011 & 0.016 & 0.010 & 0.021 & 0.021 & 0.133 & 0.025 & 0.002 & 0.030 \\
\hline$F_{y x}$ & 0.008 & 0.003 & 0.003 & 0.001 & 0.029 & 0.017 & 0.027 & 0.005 & 0.010 & 0.020 \\
\hline$F_{y y}$ & 0.130 & 0.017 & 0.042 & 0.009 & 0.035 & 0.075 & 0.067 & 0.050 & 0.167 & 0.047 \\
\hline$F_{y z}$ & 0.021 & 0.013 & 0.025 & 0.028 & 0.009 & 0.026 & 0.307 & 0.024 & 0.030 & 0.022 \\
\hline$F_{z x}$ & 0.018 & 0.011 & 0.016 & 0.010 & 0.021 & 0.021 & 0.133 & 0.025 & 0.002 & 0.030 \\
\hline$F_{z y}$ & 0.021 & 0.013 & 0.025 & 0.028 & 0.009 & 0.026 & 0.307 & 0.024 & 0.030 & 0.022 \\
\hline$F_{z z}$ & 0.039 & 0.032 & 0.061 & 0.151 & 0.026 & 0.045 & 1.586 & 0.055 & 0.015 & 0.066 \\
\hline$F_{1}$ & 0.033 & 0.010 & 0.025 & 0.004 & 0.021 & 0.030 & 0.007 & 0.026 & 0.010 & 0.032 \\
\hline$n_{1 x}$ & -0.130 & 0.020 & 0.035 & 0.040 & -0.012 & -0.021 & -0.005 & 0.188 & -0.010 & -0.001 \\
\hline$n_{1 y}$ & -0.196 & 0.860 & 0.815 & 0.981 & -0.511 & -0.495 & 0.982 & 0.671 & -0.184 & 0.839 \\
\hline$n_{1 z}$ & 0.972 & -0.510 & -0.579 & -0.190 & 0.860 & 0.869 & -0.191 & -0.717 & 0.983 & -0.543 \\
\hline$F_{2}$ & 0.130 & 0.038 & 0.076 & 0.023 & 0.034 & 0.089 & 0.291 & 0.064 & 0.059 & 0.076 \\
\hline$n_{2 x}$ & -0.372 & -0.105 & 0.104 & 0.996 & -0.160 & -0.061 & 0.995 & 0.465 & -0.996 & -0.104 \\
\hline$n_{2 y}$ & 0.918 & 0.510 & -0.579 & -0.054 & 0.850 & 0.868 & -0.014 & -0.704 & 0.089 & 0.540 \\
\hline$n_{2 z}$ & 0.136 & 0.854 & -0.809 & -0.069 & 0.503 & 0.493 & -0.099 & -0.537 & 0.006 & 0.835 \\
\hline$F_{3}$ & 0.162 & 0.144 & 0.221 & 0.157 & 0.259 & 0.509 & 1.660 & 0.115 & 0.174 & 0.423 \\
\hline$n_{3 x}$ & -0.919 & -0.994 & -0.994 & 0.078 & -0.987 & -0.998 & 0.100 & -0.865 & -0.089 & -0.995 \\
\hline$n_{3 y}$ & -0.344 & -0.036 & -0.032 & 0.187 & -0.132 & -0.043 & 0.190 & -0.232 & -0.979 & -0.057 \\
\hline$n_{3 z}$ & -0.192 & -0.100 & -0.105 & 0.979 & -0.092 & -0.049 & 0.977 & -0.445 & -0.184 & -0.087 \\
\hline$I_{1}$ & 0.325 & 0.191 & 0.322 & 0.183 & 0.314 & 0.627 & 1.958 & 0.206 & 0.243 & 0.532 \\
\hline$J_{2}$ & 0.005 & 0.005 & 0.010 & 0.007 & 0.018 & 0.068 & 0.781 & 0.002 & 0.007 & 0.046 \\
\hline
\end{tabular}

$x$ Direction is perpendicular to the bedding planes and E-F faces; $y$ direction is parallel to the bedding planes and perpendicular to B$\mathrm{D}$ faces; $z$ direction is parallel to the bedding planes and perpendicular to A-C faces; $F_{t}(t=1,2,3)$ is the principal value of the fracture tensor in $t$ direction; $n_{t x}, n_{t y}, n_{t z}(t=1,2,3)$ are the directional cosines of the principal direction $t ; I_{1}$ is the first invariant of the fracture tensor; $J_{2}$ is the second invariant of the deviatoric fracture tensor

greatest, which indicates the fracture systems of most samples are dominated by the bedding planes that are oriented approximately perpendicular to the $x$ direction. The fracture tensor based methodology may be combined with other analysis to address the anisotropic behaviors of the discontinuous rock masses induced by the fracture networks.

\section{Numerical simulation of fracture networks and validations}

\subsection{Simulation of fracture networks}

The constructed fracture networks can be imported into distinct element software 3DEC for further analysis. The following rules were used to calculate the fracture orientations and model the fractures in 3DEC:

a. For a fracture plane, when more than one trace can be observed from CT slices, it is possible to calculate its true dip and dip direction. These fractures, which maybe either bedding planes or cleats, were simplified as equivalent squares in 3DEC;

b. For a fracture plane, when only one trace is available from the vertical CT slices, only the apparent dip angle and the apparent dip direction angle of the fracture plane can be estimated for the fracture plane. These fractures, which also may belong to either the bedding planes or cleats, were idealized as equivalent rectangles in 3DEC; 
Fig. 7 Concept of fictitious joint in 2-D: a Inclination angle of real joint $\leq 45^{\circ}$. b Inclination angle of real joint $>45^{\circ}$

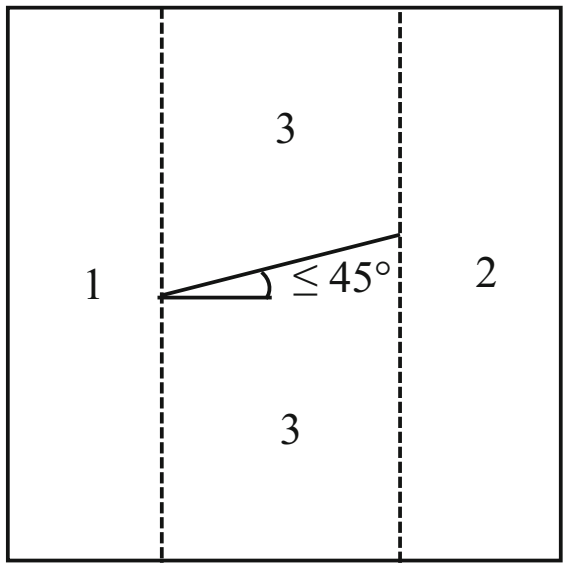

(a)

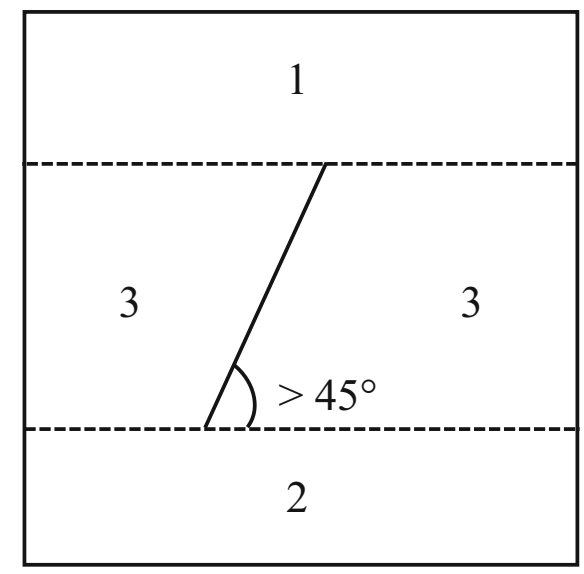

(b)
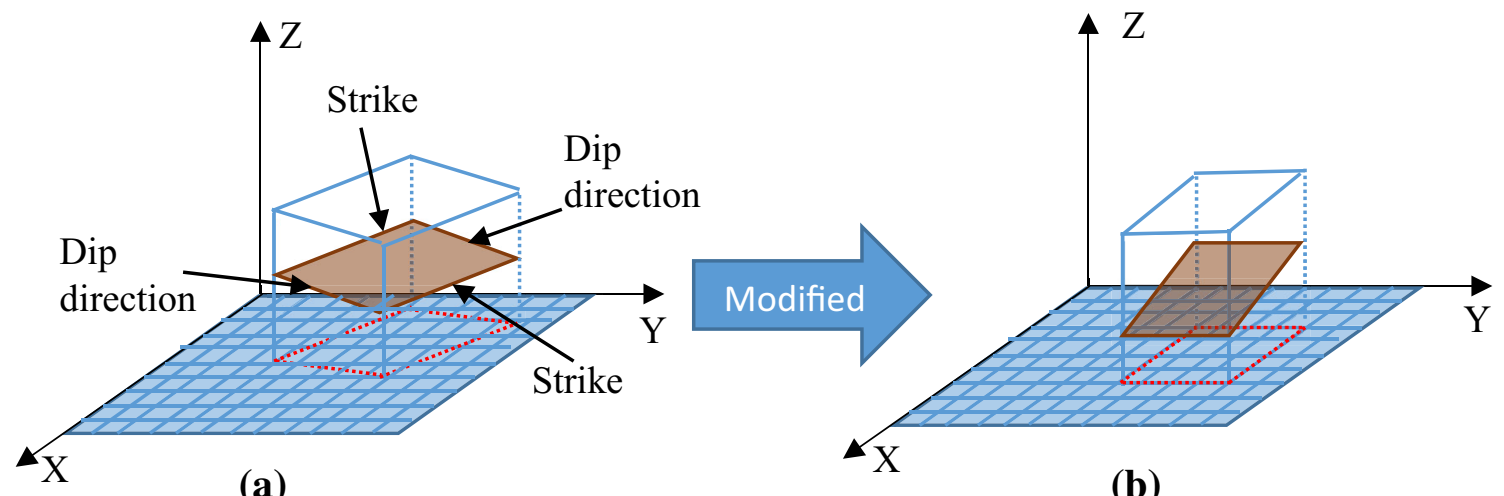

(a)

Fig. 8 Schematic diagram of fictitious joint in 3-D (Dip angle $\leq 45^{\circ}$ ): a Original framework. b Modified framework

c. For a cleat, when only one trace is available from the horizontal CT slices, only the strike of the fracture planes can be estimated. Therefore, for these fracture planes, the dip angle was assumed to be $90^{\circ}$. These fractures were also represented as equivalent rectangles in $3 \mathrm{DEC}$.

In order to make any stress analysis using either the UDEC or 3DEC, the problem domain should be first discretized into polygons in 2-D or polyhedral in 3-D. Since almost all the discontinuities in the cubic coal blocks are non-persistent and 3DEC only allows persistent joint cutting through the blocks, the fictitious joint procedure proposed by Kulatilake et al. (1992) and Wang and Kulatilake (1993) was implemented to make the initial attempt of building up the geometric model. With this method, the fictitious joints are created in such a way to discretize the rock blocks into polygons in 2-D or polyhedral in 3-D when those are combined with the actual joints. The concept of the fictitious joint in 2-D is shown in Fig. 7. The dash lines represent the fictitious joints while the solid lines are real joints. First, two fictitious joints are created as shown in Fig. 7 to discretize the rock block into polygonal blocks 1,2 and 3 . Then by hiding blocks 1 and 2 the real joint is placed in block 3 as shown in Fig. 7 to separate block 3 into two polygons. Depending on the inclination angle the real joint makes with the horizontal line, the fictitious joints are set to be either vertical (inclination angle $\leq 45^{\circ}$ ) or horizontal (inclination angle $>45^{\circ}$ ) to reduce the stress concentration which may happen around the sharp 


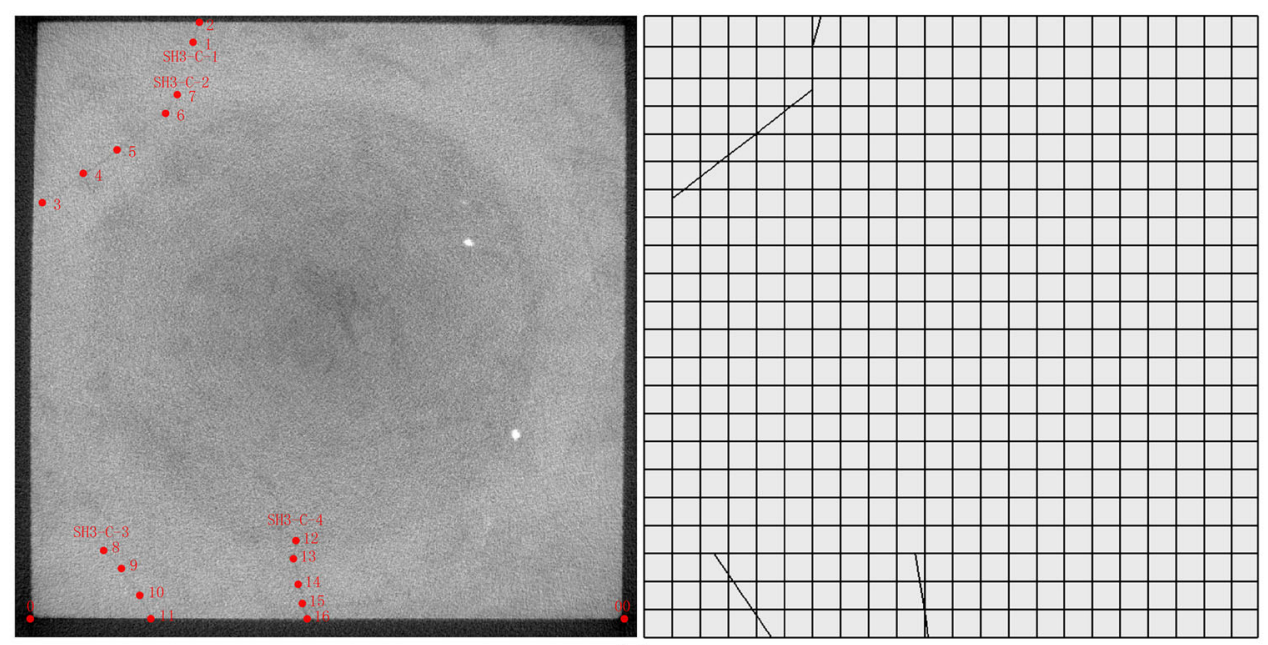

(a)

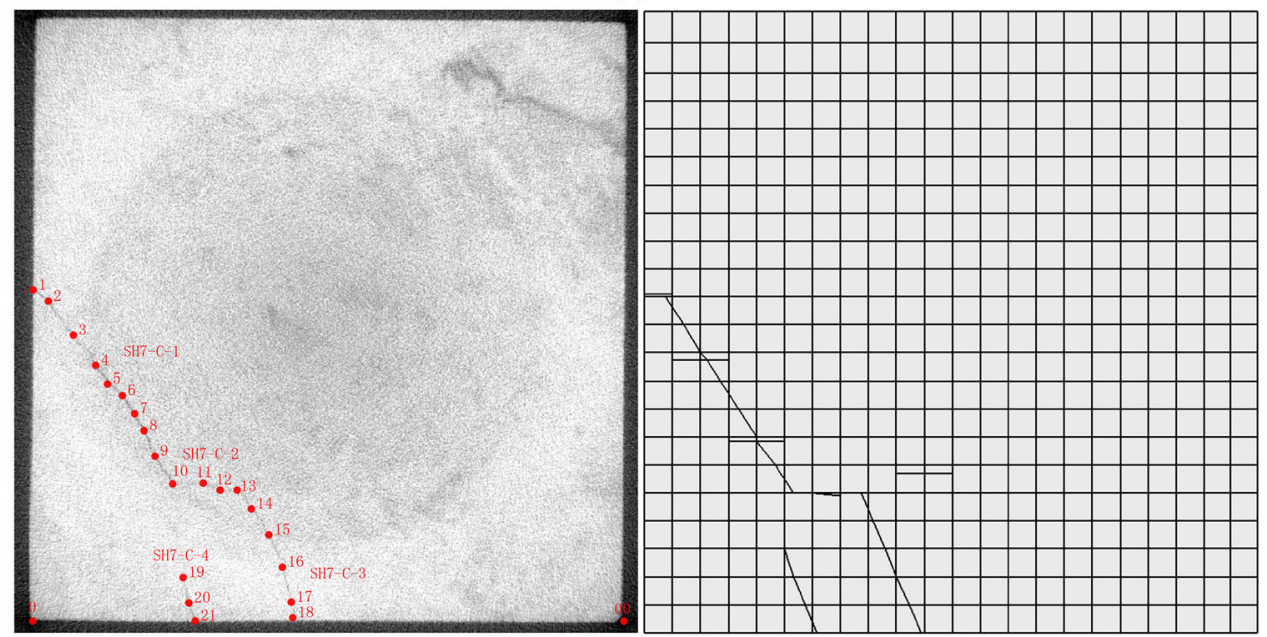

(b)

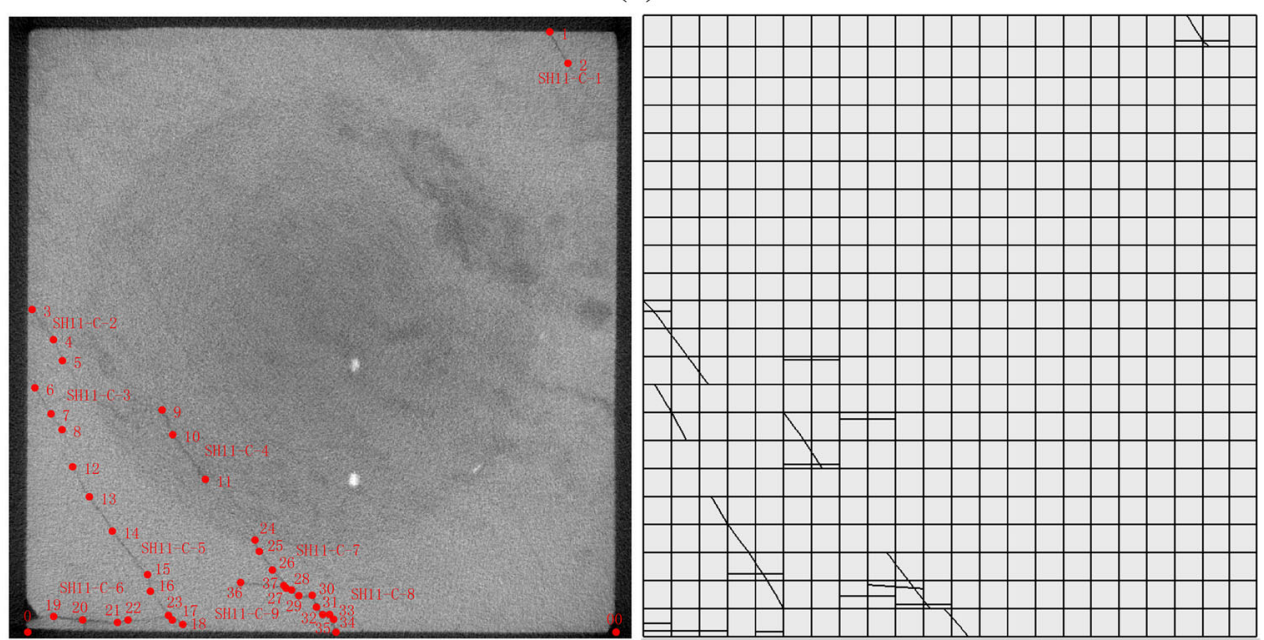

(c) 
4 Fig. 9 Comparison between the numerical cubic coal block and physical cubic coal block with respect to some cross sections subparallel to the bedding planes for CB60 as an example: a $\mathrm{H}_{\mathrm{h}}=20 \mathrm{~mm}$. b $\mathrm{H}_{\mathrm{h}}=60 \mathrm{~mm}$. c $\mathrm{H}_{\mathrm{h}}=100 \mathrm{~mm}$

corners during the numerical calculations. In a 3-D case, a non-persistent real joint should first be simplified as a rectangular fracture plane with the same area. Then, the first pair of fictitious joints should be two vertical planes passing on the two opposite sides along the dip direction of the rectangular fracture plane. The second pair of fictitious joints should pass on the other two opposite sides parallel to the strike of the rectangular fracture plane. Similar to a 2-D case, depending on the dip angle of the real joint, the second pair of fictitious joints should be set either vertical (dip angle $\leq 45^{\circ}$ ) or horizontal (dip angle $>45^{\circ}$ ) to reduce the stress concentration which may happen around the sharp corners during the numerical calculations (see Fig. 8a) for a case of joint dip angle $\leq 45^{\circ}$ as an example). The Matlab software package was first used to write a code to transform the fracture data into a format easily accessible to 3DEC. A FISH (built-in programming language in 3DEC) code was written to introduce all the fractures constructed from the CT scans into the cubic coal block using the fictitious joint framework.

According to the original fictitious joint framework, four fictitious joints are needed whenever a nonpersistent joint is inserted into a rock block. Therefore, application of this technique results in a large number of rock blocks in case of the fracture networks with a large quantity of non-persistent joints. Because of the high numbers of pre-existing non-persistent discontinuities involved in the cubic coal blocks encountered in this research, it took a considerable amount of time to build up the geometric model (more than $24 \mathrm{~h}$ ). It was found very time consuming to mesh the problem domain and perform the stress analysis by 3DEC using the original fictitious joint framework. To reduce the numerical effort to an acceptable range, a modified fictitious joint framework was developed to cut down the number of the fictitious joints needed. As shown in Fig. 8b), the $110 \mathrm{~mm}$ cubic coal block is pre-cut by three sets of fictitious joints perpendicular to $\mathrm{X}, \mathrm{Y}$ and $\mathrm{Z}$ axes. Simultaneously, the three coordinate planes, $\mathrm{XY}$ plane, $\mathrm{YZ}$ plane and $\mathrm{XZ}$ plane, are divided into a certain number of square grids. The spacing of each fictitious joint set can be selected based on the acceptable accuracy. For different fictitious joint sets, the spacing can be set different or the same. In this research, the spacing of the fictitious joints was set equal to the spacing of the CT slices. Then, the coordinate plane providing the maximum projection area of a given fracture plane can be found. This maximum projection area is reshaped to find an equivalent rectangular area coinciding with the grid lines and providing the closest area to the maximum projection area (adjusted maximum projection, see Fig. 8b). The center of this equivalent rectangular area should coincide with the projection of the center of the fracture plane. The four fictitious joints of this particular fracture plane are perpendicular to the aforementioned specific coordinate plane and passing at the four boundaries of the adjusted maximum projection. The fracture plane with the same orientation can then be placed into the prismatic block formed by the fictitious joints. The idea of using the maximum projection area is to reduce the stress concentration around the sharp corners, similar to the dip angle based principle in the original framework. With this modified procedure, a rhomboid fracture plane is obtained with the same orientation and almost the same area as the original rectangular fracture plane. The advantage of this modified fictitious joint framework is that the three sets of fictitious joints can serve as the common fictitious joints for any number of non-persistent joints. The reduced number of the fictitious joints will consume much less numerical stress analysis effort than that of the original fictitious joint procedure (reducing from more than $24 \mathrm{~h}$ to about $5 \mathrm{~min}$ ). The modified fictitious joint framework keeps the two most important parameter values of a joint: joint orientation and joint size the same to a great extent. This modified fictitious joint framework was realized through modifying the aforementioned Matlab code and FISH code for the original framework.

\subsection{Validation of fracture networks}

To validate the built-up geometric model using the modified fictitious joint framework, comparisons were made between some cross sections of the built-up geometric model and the corresponding CT scanning slices. As shown in Figs. 9 and 10, a good agreement was found for sample CB60 between the fracture 


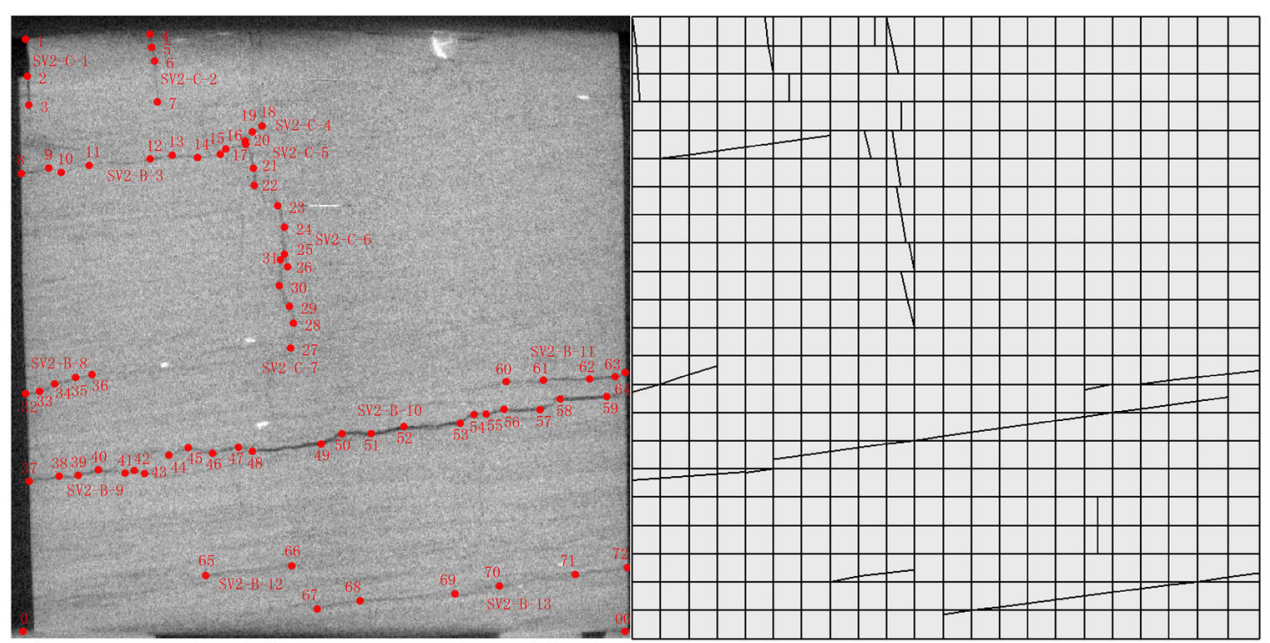

(a)

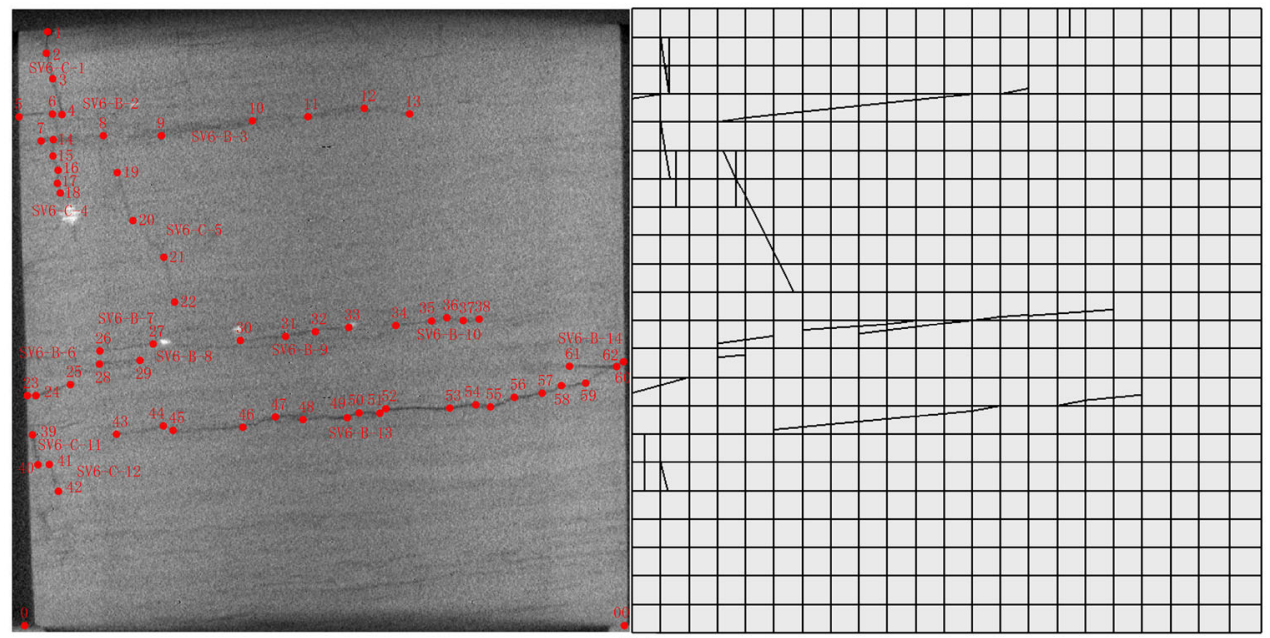

(b)

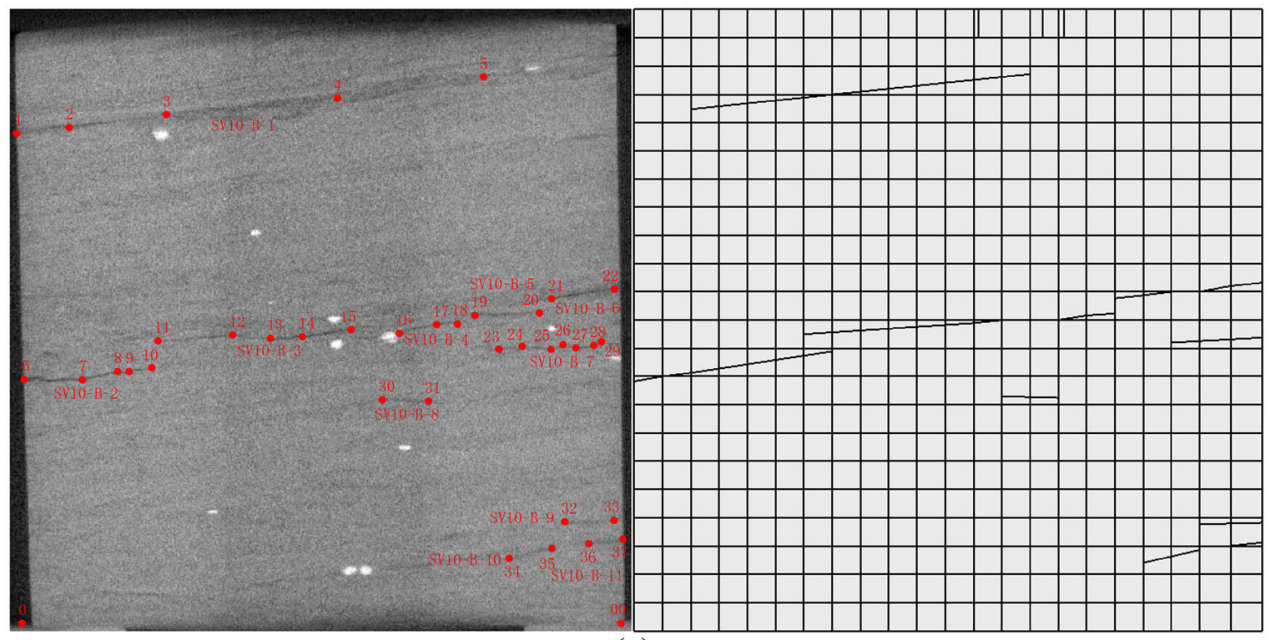

(c) 
4Fig. 10 Comparison between the numerical cubic coal block and physical cubic coal block with respect to some cross sections approximately perpendicular to the bedding planes for CB60 as an example: a $\mathrm{H}_{\mathrm{v}}=10 \mathrm{~mm}$. b $\mathrm{H}_{\mathrm{v}}=50 \mathrm{~mm}$. c $\mathrm{H}_{\mathrm{v}}=90 \mathrm{~mm}$

network of the numerical cubic coal block and that of the physical cubic coal block. In Figs. 9 and 10, the horizontal and vertical persistent lines are fictitious joints, whereas the inclined short lines represent the non-persistent pre-existing joints. Please note that discrepancies may be found in a few areas because the used modified fictitious joint framework simplified the irregular polygonal shape of the real non-persistent joints to improve the computational efficiency. This might have resulted in shifting some joints slightly. Considering the possible variability arising from geomechanical parameters in numerical analysis, the geometric models built-up by this procedure are satisfactory and the minor discrepancies are acceptable. The created numerical coal block and fracture networks for CB60 are shown in Figs. 11 and 12 as examples.

In the application of the modified fictitious joint procedure, the shape of each real joint was changed from a rectangle to a rhomboid. If the shape change is too much, a possibility exists for sharp corners of the rhomboid to cause some problems in the numerical calculations. Because the natural fracture networks in coal masses are approximately orthogonal, the axes of the coordinate system can be selected approximately along the normal directions of the three approximately orthogonal joint sets. Thus, the shape change from a rectangle to a rhomboid is limited to a certain extent and the possible stress concentration caused by the shape change is controlled within an acceptable range. Please note that most of the fractures constructed from the CT scans were obtained through the single traces on the horizontal or vertical CT slices. Because of the assumptions used in the proposed construction procedure, the shapes of these fractures were not changed by the modified fictitious joint procedure. The fractures that experienced a shape change were the ones constructed from the multiple traces, which only occupied a small proportion of the whole fracture network in this research. This modified fictitious joint procedure may also be used to build up the geometric model of other rock masses having approximately orthogonal fracture systems. For the rock masses having non-orthogonal fracture systems, this procedure need to be used with great caution and systematic verifications.

To evaluate the calculation accuracy of the modified fictitious joint procedure, two numerically
Fig. 11 Numerical model of the jointed cubic coal block of sample CB60 as an example

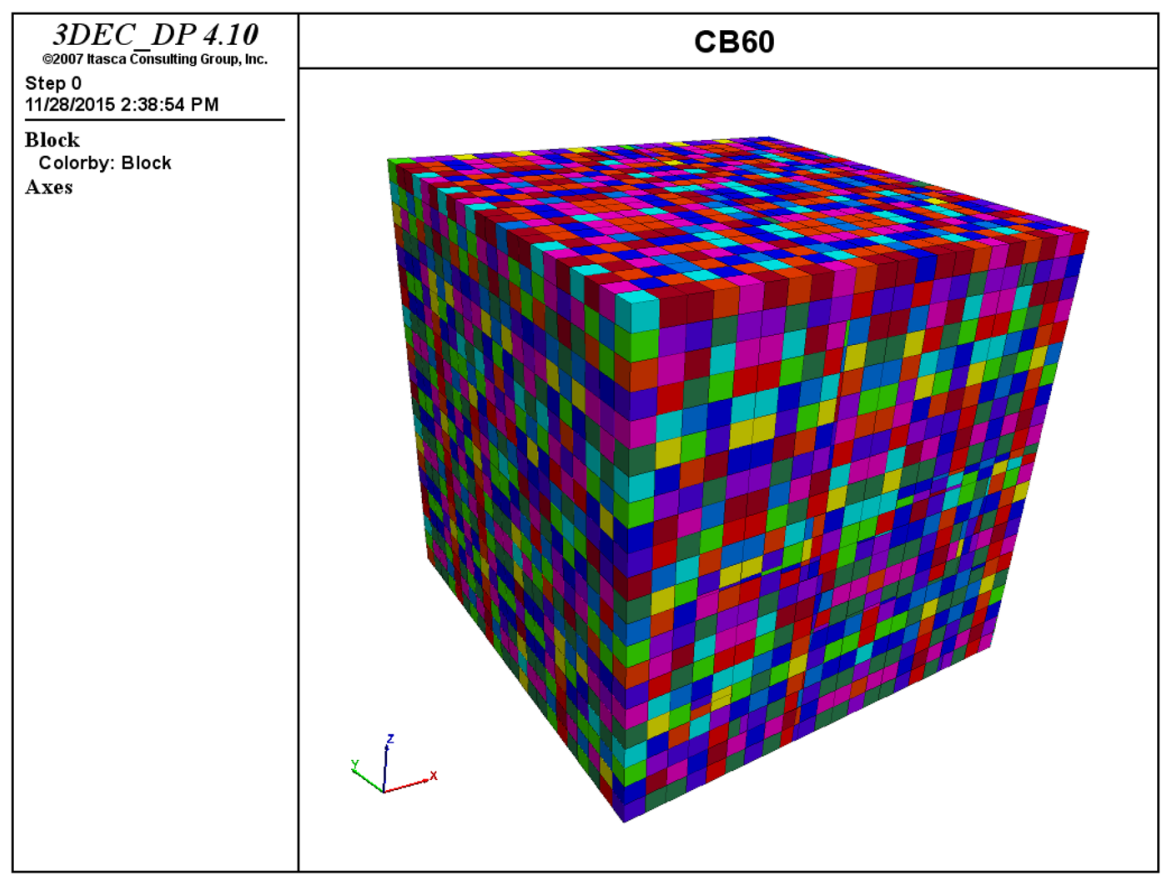


Fig. 12 Fracture network of sample CB60 including fictitious and real joints as an example

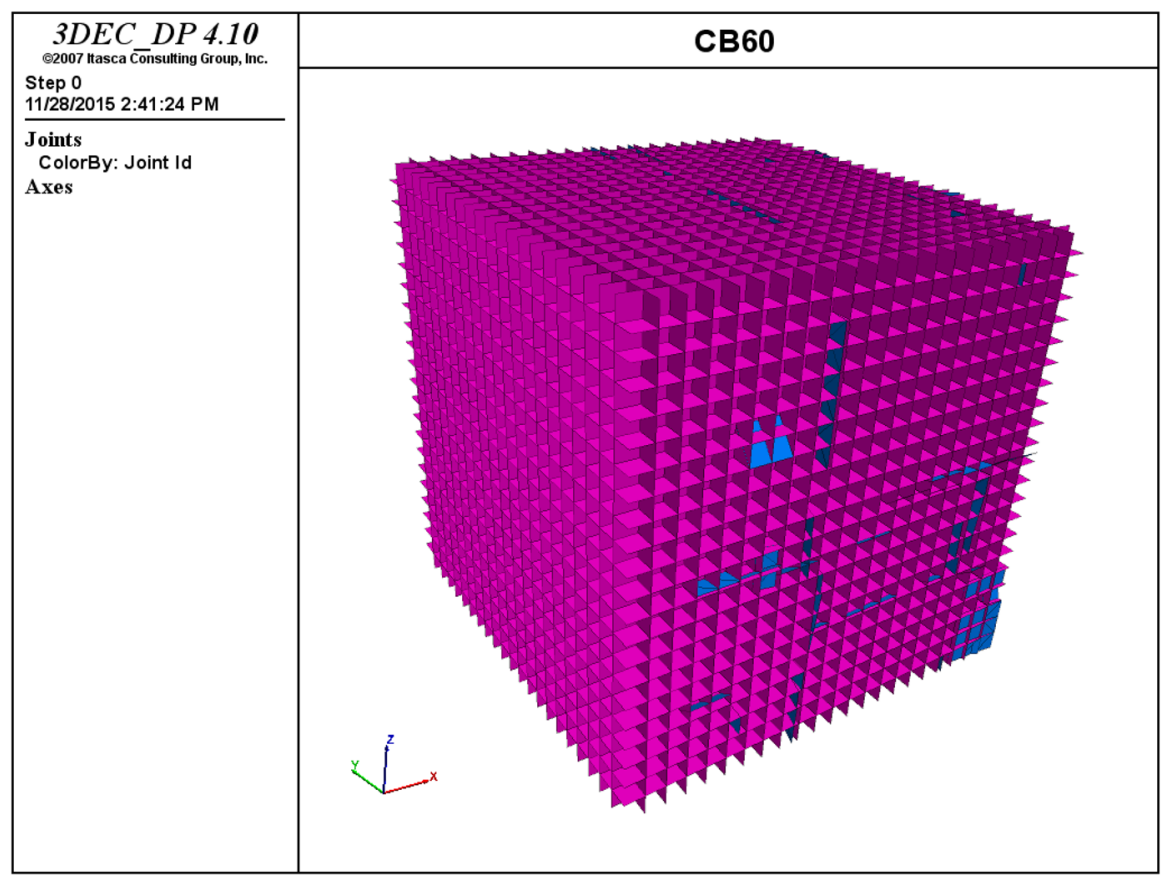

simulated jointed cubic coal blocks with all eleven fractures of sample CB48 constructed from the multiple traces were created by the original and modified fictitious joint procedures, respectively. The two models were subjected to the polyaxial compression tests with the numerical parameter values given in Table 4 under seven different confining stresses (the two directional confining stresses are the same). The numerical parameters listed in Table 4 were obtained from a systematic calibration and validation using the laboratory polyaxial compression test results. The geomechanical parameter values of the fictitious joints were calculated from the geomechanical property values of intact coal according to the guidelines provided in Kulatilake et al. (1992). The comparison of the jointed cubic coal block strengths obtained from the two numerical models is shown in Fig. 13. The obtained strength differences between the two procedures are highly acceptable.

\section{Example of implementing the proposed procedure}

The proposed procedure was implemented in a numerical investigation to show the influence of the

Table 4 Geomechanical parameter values of intact coal, fictitious joints and real joints used in the calibrated numerical model

\begin{tabular}{lllllc}
\hline Intact coal (Mohr-Coulomb plasticity) & Fictitious joints (Coulomb slip model) & \multicolumn{2}{l}{ Real coal joints (continuously yielding model) } \\
\hline Density $(\rho)$ & $1200 \mathrm{~kg} / \mathrm{m}^{3}$ & $\mathrm{JKN}$ & $411.99 \mathrm{GPa} / \mathrm{m}$ & $\mathrm{JKN}_{0}$ & $5.0375 \times 10^{4} \mathrm{~m}^{-1}$ \\
Young's modulus $(E)$ & $4.12 \mathrm{GPa}$ & $\mathrm{JKS}$ & $147.14 \mathrm{GPa} / \mathrm{m}$ & $\mathrm{Min} \mathrm{JKN}$ value & $0 \mathrm{~Pa} / \mathrm{m}$ \\
Poisson's ratio $(v)$ & 0.40 & Joint cohesion $(j c)$ & $6.95 \mathrm{MPa}$ & $\mathrm{Max} \mathrm{JKN}$ value & $2.5 \times 10^{12} \mathrm{~Pa} / \mathrm{m}$ \\
Cohesion $\left(c_{0}\right)$ & $6.95 \mathrm{MPa}$ & Joint friction angle $\left(j \varphi_{1}\right)$ & $28.64^{\circ}$ & $\mathrm{JKS}_{0}$ & $9.42 \times 10^{2} \mathrm{~m}^{-1}$ \\
Friction angle $(\varphi)$ & $28.64^{\circ}$ & Joint tensile strength $(j t)$ & $1.21 \mathrm{MPa}$ & Min JKS value & $0 \mathrm{~Pa} / \mathrm{m}$ \\
Tensile strength $\left(\sigma_{t}\right)$ & $1.21 \mathrm{MPa}$ & & & Max JKS value & $5 \times 10^{10} \mathrm{~Pa} / \mathrm{m}$ \\
& & & & Joint friction angle $\left(j \varphi_{2}\right)$ & $35^{\circ}$ \\
& & & & Joint roughness $(r)$ & $3 \mathrm{~mm}$ \\
\hline
\end{tabular}


Fig. 13 Comparison of the block strengths obtained from the original fictitious joint procedure and modified fictitious joint procedure

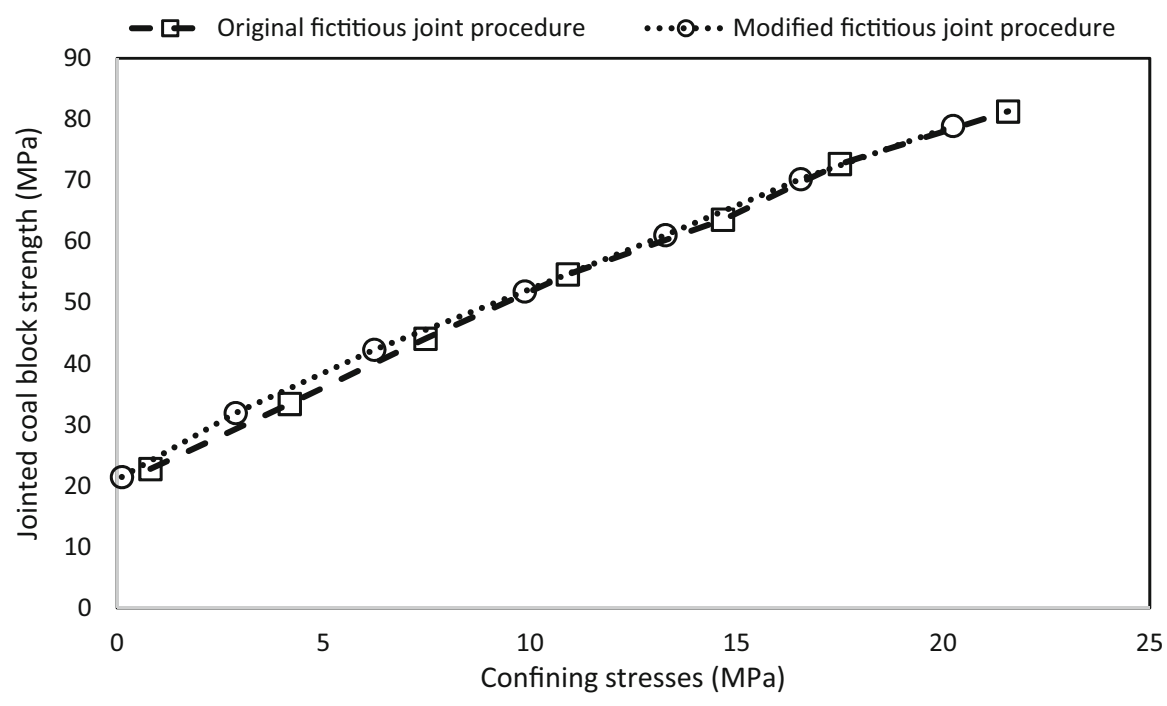

confining stresses and fracture networks on the jointed coal mass strength (JCMS). Those samples subjected to CT scanning were also tested under polyaxial compression conditions. JCMS values of the cubic coal blocks under different confining stresses were obtained through laboratory polyaxial compression tests. These laboratory values are denoted by $\mathrm{JCMS}_{\mathrm{L}}$. The fracture network of one cubic coal block CB22 was detected, constructed, quantified and numerically simulated using the proposed procedure. Through a trial and error calibration procedure, the numerical parameters needed in 3DEC were obtained and are listed in Table 4. Another ten samples were selected to represent the cubic coal block populations with respect to spectrums of confining stresses, fracture networks and JCMS values. The same procedure was implemented on these ten cubic coal blocks to validate the numerical parameters in Table 4. The validation results obtained are shown in Table 5. From Table 5, the deviation of JCMS from $\mathrm{JCMS}_{\mathrm{L}}$ for nine out of the

Table 5 Summary of validation results for the selected eleven samples

\begin{tabular}{|c|c|c|c|c|c|c|c|c|c|}
\hline Sample & $\sigma_{y}(\mathrm{MPa})$ & $\sigma_{x}(\mathrm{MPa})$ & $F_{x x}$ & $F_{y y}$ & $F_{z z}$ & $F_{x x}+F_{y y}$ & $\mathrm{JCMS}_{\mathrm{L}}(\mathrm{MPa})$ & JCMS (MPa) & Dev. (\%) \\
\hline $\mathrm{CB} 22^{\mathrm{a}}$ & 7.101 & 7.101 & 0.323 & 0.118 & 0.152 & 0.441 & 52.28 & 50.75 & -2.9 \\
\hline CB24 & 4.734 & 2.367 & 0.410 & 0.007 & 0.074 & 0.418 & 36.42 & 36.54 & 0.3 \\
\hline CB41 & 0 & 2.367 & 0.507 & 0.076 & 0.052 & 0.584 & 23.39 & 25.22 & 7.8 \\
\hline CB6 & 0 & 7.101 & 0.507 & 0.075 & 0.045 & 0.582 & 32.74 & 29.49 & -9.9 \\
\hline CB4 & 0 & 0 & 0.023 & 0.009 & 0.151 & 0.033 & 15.78 & 20.04 & 27.0 \\
\hline CB48 & 4.734 & 2.367 & 1.744 & 0.076 & 0.072 & 1.820 & 35.69 & 33.71 & -5.5 \\
\hline CB14 & 7.101 & 7.101 & 0.578 & 0.117 & 0.053 & 0.695 & 47.75 & 50.00 & 4.7 \\
\hline CB60 & 4.734 & 2.367 & 0.833 & 0.072 & 0.081 & 0.905 & 35.38 & 34.71 & -1.9 \\
\hline CB57 & 7.101 & 0 & 0.124 & 0.026 & 0.028 & 0.150 & 23.38 & 28.22 & 20.7 \\
\hline CB3 & 4.734 & 9.468 & 0.219 & 0.042 & 0.061 & 0.262 & 46.99 & 50.45 & 7.4 \\
\hline $\mathrm{CB} 2$ & 0 & 4.734 & 0.142 & 0.017 & 0.032 & 0.159 & 27.19 & 29.33 & 7.9 \\
\hline
\end{tabular}

The values in bold are $<10$

$\mathrm{JCMS}_{\mathrm{L}}$-is the jointed coal mass strength obtained from the laboratory tests, JCMS—is the corresponding jointed coal mass strength obtained through numerical modeling, Dev.- - is the deviation of JCMS from JCMS $\mathrm{L}_{\mathrm{L}}$

${ }^{\text {a }}$ Is the sample used for the calibration of geomechanical parameters 
eleven samples is $<10 \%$, which means the numerical parameters in Table 4 were successfully validated.

To provide an example of implementing the proposed procedure, three samples (CB2, CB24, and CB48) are selected from Table 5 to perform numerical analyses to study the influence of the fracture network and confining stresses on the JCMS values. The following two diagrams Figs. 14 and 15 are plotted based on the obtained numerical calculations. In both figures, the $F_{x x}+F_{y y}$ values (the summation of the fracture tensor components in the two perpendicular directions to the loading direction) increase from the top line to the bottom line. According to the two figures, the following two statements can be made from the observations:

a. When the confining stress system is the same, the higher the summation of the fracture tensor components in the two perpendicular directions to the loading direction, the lower the JCMS value in the loading direction;

b. When the summation of the fracture tensor components in the two perpendicular directions to the loading direction is the same, the higher the confining stress, the higher the JCMS value in the loading direction.
Fig. 14 Relations obtained between JCMS and $\sigma_{x}$ for eight fracture network setups with $\sigma_{y}=7.101 \mathrm{MPa}$
Fig. 15 Relations obtained between JCMS and $\sigma_{y}$ for nine fracture network setups with $\sigma_{x}=2.367 \mathrm{MPa}$
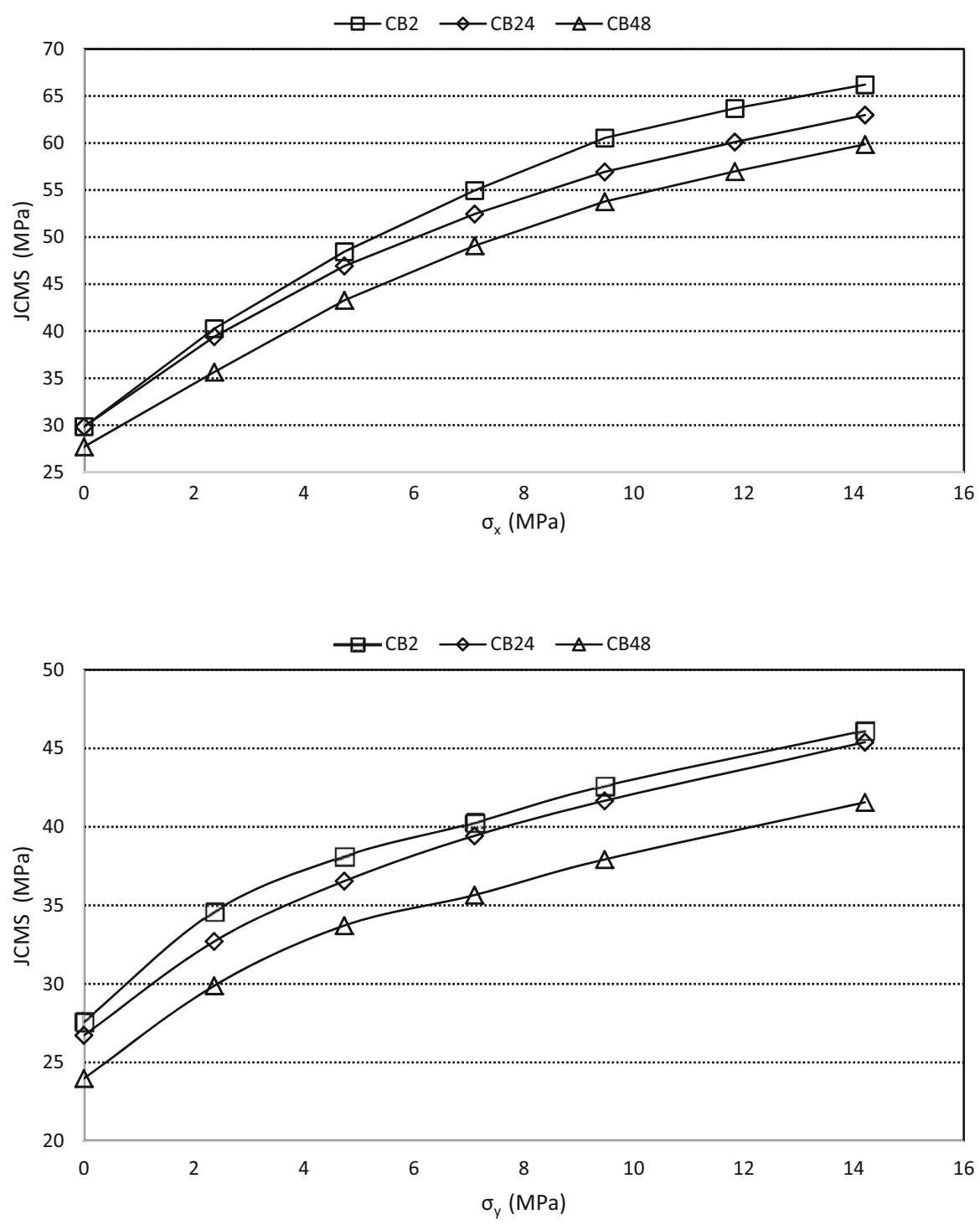


\section{Discussion}

As mentioned previously, to reduce the stress concentrations at the sharp corners caused by fracture shape change during the implementation of the modified fictitious joint procedure, it is suggested to select the axes of the coordinate system approximately along the normal directions of the pre-existing three orthogonal joint sets. Generally, the stress boundary conditions are provided in a different coordinate system, in which three axes coincide with three principal directions of the in situ stresses. To implement the modified fictitious joint procedure in a numerical analysis, the boundary stresses need to be transformed to the stresses corresponding to the selected coordinate system, which most likely contain the shear components.

\section{Summary and conclusions}

A framework incorporating fracture observation, construction, quantification and simulation was proposed in this paper. The proposed framework was implemented in a numerical investigation to show the influence of fracture networks and confining stresses on the jointed coal mass strength. The following are the main features of this framework:

a. A fracture construction procedure was developed to obtain the geometric information of three orthogonal joint sets in a coal mass (bedding planes, face cleats and butt cleats) from the laboratory CT scanning test;

b. Fracture tensor based methodology was suggested to provide a comprehensive assessment for the fracture network in a coal mass. With this method, the important feature (number of joint sets, joint density, joint orientation and joint size) of the fractures can be included into a tensor based parameter;

c. A modified fictitious joint procedure was proposed to accommodate a large quantity of nonpersistent joints. Because of the reduction of the number of fictitious joints, the numerical effort was much less than that using the original fictitious joint procedure.

Acknowledgments The research was funded by the Centers for Disease Control and Prevention (Contract No. 200-201139886), Chinese Natural Science Research Council (Project No.
11572344) and the State Key Laboratory for Geomechanics and Deep Underground Engineering, China University of Mining and Technology (Contract No. SKLGDUEK1416).

\section{References}

Andersson J, Dverstorp B (1987) Conditional simulations of fluid flow in three-dimensional networks of discrete fractures. Water Resour Res 23(10):1876-1886

Baecher GB (1980) Progressively censored sampling of rock joints traces. Math Geol 12(1):33-40

Bidgoli MN, Jing L (2014) Anisotropy of strength and deformability of fractured rocks. J Rock Mech Geotech Eng 6(2):156-164

Bidgoli MN, Zhao Z, Jing L (2013) Numerical evaluation of strength and deformability of fractured rocks. J Rock Mech Geotech Eng 5(6):419-430

Bulut F, Tüdeş Ş (1996) Determination of discontinuity traces on inaccessible rock slopes using electronic tacheometer: an example from the Ikizdere (Rize) Region, Turkey. Eng Geol 44(1):229-233

Cacus MC, Ledoux E, De Marsily G, Tille B, Barbreau A, Durand E, Feuga B, Peaudecerf P (1990) Modeling of fracture flow with a stochastic discrete fracture network: calibration and validation-1, the flow model. Water Resour Res 26(3):479-489

Coe JA (1995) Close-range photogrammetric geological mapping and structural analysis. Master Thesis, Colorado School of Mines, USA

Damjanac B, Cundall P (2016) Application of distinct element methods to simulation of hydraulic fracturing in naturally fractured reservoirs. Comput Geotech 71:283-294

Edelbro C (2003) Rock mass strength-a review. http://epubl. luth.se/1402-1536/2003/16/LTU-TR-0316-SE.pdf. Accessed 16 May 2015

Edelbro C, Sjöberg J, Nordlund E (2006) A quantitative comparison of strength criteria for hard rock masses. Tunn Undergr Sp Tech 22(1):57-68

Feng Q, Röshoff K (2015) A survey of 3D laser scanning techniques for application to rock mechanics and rock engineering. In: Ulusay R (ed) The ISRM suggested methods for rock characterization, testing and monitoring: 2007-2014. Springer International Publishing, New York, pp 265-293

Hagan TO (1980) A case for terrestrial photogrammetry in deepmine rock structure studies. Int $\mathrm{J}$ Rock Mech Min 17(4):191-198

Harrison JP (1993) Improved analysis of rock mass geometry using mathematical and photogrammetric methods. Ph.D. Thesis, Imperial College, London

Kulatilake PHSW, Panda BB (2000) Effect of block size and joint geometry on jointed rock hydraulics and REV. J Eng Mech ASCE 126(8):850-858

Kulatilake PHSW, Shu B (2015) Prediction of rock mass deformations in three dimensions for a part of an open pit mine and comparison with field deformation monitoring data. Geotech Geol Eng 33:1551-1568

Kulatilake PHSW, Wu TH (1984) Estimation of mean trace length of discontinuities. Rock Mech Rock Eng 17:215-232 
Kulatilake PHSW, Ucpirti H, Wang S, Radberg G, Stephansson O (1992) Use of the distinct element method to perform stress analysis in rock with non-persistent joints and to study the effect of joint geometry parameters on the strength and deformability of rock masses. Rock Mech Rock Eng 25(4):253-274

Kulatilake PHSW, Wang S, Stephansson O (1993) Effect of finite size joints on the deformability of jointed rock in three dimensions. Int J Rock Mech Min 30(5):479-501

Kulatilake PHSW, Um JG, Wang M, Escandon RF, Narvaiz J (2003) Stochastic fracture geometry modeling in 3-D including validations for a part of Arrowhead East Tunnel, California. USA. Eng geol 70(1):131-155

Li SJ, Feng XT, Wang CY, Hudson JA (2012) ISRM suggested method for rock fractures observations using a borehole digital optical televiewer. In: Ulusay R (ed) The ISRM suggested methods for rock characterization, testing and monitoring: 2007-2014. Springer International Publishing, New York, pp 159-168

Men XX, Tang CA, Wang SY, Li YP, Yang T, Ma TH (2013) Numerical simulation of hydraulic fracturing in heterogeneous rock: the effect of perforation angles and bedding plane on hydraulic fractures evolutions. In: ISRM international conference for effective and sustainable hydraulic fracturing. International Society for Rock Mechanics, pp 491-511

Oda M (1982) Fabric tensor for discontinuous geological materials. Soils Found 22(4):96-108

Pahl PJ (1981) Estimating the mean length of discontinuity traces. Int J Rock Mech Min 18(3):221-228
Panda BB, Kulatilake PHSW (1999) Relations between fracture tensor parameters and permeability tensor parameters for discontinuous rock. ASCEJ Eng Mech 125(1):51-59

Priest SD (1993) Discontinuity analysis for rock engineering. Chapman and Hall, London

Priest SD, Hudson JA (1981) Estimation of discontinuity spacing and trace length using scanline surveys. Int J Rock Mech Min 18(3):183-197

Slob S, Van Knapen B, Hack R, Turner K, Kemeny J (2005) Method for automated discontinuity analysis of rock slopes with three-dimensional laser scanning. Transp Res Rec J Transp Res Board 1913:187-194

Wang S, Kulatilake PHSW (1993) Linking between joint geometry models and a distinct element method in three dimensions to perform stress analyses in rock masses containing finite size joints. Soils Found 33(4):88-98

Wang M, Kulatilake PHSW, Um J, Narvaiz J (2002) Estimation of REV size and three dimensional hydraulic conductivity tensor for a fractured rock mass through a single well packer test and discrete fracture fluid flow modeling. Int $\mathrm{J}$ Rock Mech Min 39(7):887-904

Wang XL, Shi F, Liu H, Wu HA (2016) Numerical simulation of hydraulic fracturing in orthotropic formation based on the extended finite element method. J Nat Gas Sci Eng 33:56-69

Wu Q, Kulatilake PHSW (2012) REV and its properties on fracture system and mechanical properties, and an orthotropic constitutive model for a jointed rock mass in a dam site in China. Comput Geotech 43:124-142 